\title{
SOCIAL LEARNING THEORY ON FACTORS ASSOCIATED WITH DENTAL CARIES AMONG MENTALLY DISABLED SCHOOL CHILDREN IN SURAKARTA, CENTRAL JAVA
}

\author{
Anggia Rahmah Nursani ${ }^{1)}$, Bhisma Murti ${ }^{1)}$, Eti Poncorini Pamungkasari²) \\ ${ }^{1)}$ Masters Program in Public Health, Sebelas Maret University \\ 2)Faculty of Medicine, Sebelas Maret University
}

\begin{abstract}
Background: Mentally disabled adolescents have limitations that make them are at greater risk of dental caries. There is a lack of studies on the factors associated with dental caries in adolescents using Social Learning Theory. This study aimed to determine factors associated with dental caries among mentally disabled adolescents using Social Learning Theory.

Subjects and Method: This was an analytic observational study using cross-sectional design. The study was conducted at several special schools for disabled children (SLB) Surakarta, including: SLB C Setya Darma, SLB C YPSLB, SLB CG YPPCG Bina Sejahtera, and SLB C1 YSSD, in Surakarta, Central Java, from June to July 2017. A sample of 150 mentally disabled school children were selected for this study by purposive sampling. The dependent variable was caries dental status. The independent variables were parenting time, maternal oral health knowledge, maternal attitude towards oral health, maternal oral hygiene practice, child oral hygiene practice, maternal sweet food intake, child sweet food intake. Dental caries status was measured by decay, missing, filled-teeth (DMF-T) index. The other data were collected by questionnaire. The data were analyzed by path analysis.

Results: Dental caries was directly and positively associated with sweet food intake $(b=0.27, \mathrm{SE}=0.09, \mathrm{p}=0.002)$, poor child oral hygiene practice $(b=$ $0.09, \mathrm{SE}=0.04, \mathrm{p}=0.018)$, and poor maternal oral health knowledge $(\mathrm{b}=$ $0.36, \mathrm{SE}=0.10, \mathrm{p}<0.001)$. Maternal oral hygiene practice was associated with maternal attitude towards oral health $(\mathrm{b}=0.33, \mathrm{SE}=0.13, \mathrm{p}=0.012)$ and maternal oral health $(b=0.18, p<0.001)$. Child oral hygiene practice was associated with maternal oral hygiene practice $(b=0.33, \mathrm{SE}=0.06, p=0.012)$, maternal oral health knowledge $(\mathrm{b}=0.91, \mathrm{SE}=0.18, \mathrm{p}<0.001)$, and parenting time $(b=1.39, \mathrm{SE}=0.18, \mathrm{p}<0.001)$.

Conclusion: Dental caries is associated with sweet food intake, poor child oral hygiene practice, and poor maternal oral health knowledge. Maternal knowledge, attitude, and practice in oral hygiene have an important role on dental caries in mentally disabled adolescents.
\end{abstract}

Keywords: dental caries, adolescents, maternal oral health practice, social learning theory

Correspondence: Anggia Rahmah Nursani. Masters Program in Public Health, Sebelas Maret University, Jl. Ir. Sutami 36A, Surakarta, Central Java. Email: anggiarahmahn23@gmail.com. 